\title{
The Emergence of the Local Moment Molecular Spin Transistor
}

\author{
Guanhua Hao, ${ }^{1}$ Ruihua Cheng ${ }^{2}$ and P. A. Dowben ${ }^{1}$ \\ ${ }^{1}$ Department of Physics and Astronomy, University of Nebraska, Lincoln, Nebraska, 68588- \\ 0299, U.S.A. \\ ${ }^{2}$ Physics Department, Indiana University Purdue University-Indianapolis, $402 \mathrm{~N}$ Blackford Str. \\ LD154, Indianapolis IN 46202, U.S.A.
}

\begin{abstract}
Local moment molecular systems have now been used as the conduction channel in gated spintronics devices, and some of these three terminal devices might even be considered molecular spin transistors. In these systems, the gate voltage can be used to tune the molecular level alignment, while applied magnetic fields have an influence on the spin state, altering the magnetic properties, and providing insights to the magnetic anisotropy. More recently, the use of molecular spin crossover complexes, as the conduction channel, has led to devices that are both nonvolatile and have functionality at higher temperatures, indeed some devices have now been demonstrated to work at room temperature. Here, several molecular transistors, including those claiming to use single molecule magnets, are reviewed.
\end{abstract}

Keywords: molecular spintronics, spin transistors, molecular electronics, magneto-electric coupling

This is the author's manuscript of the article published in final edited form as:

Hao, G., Cheng, R., \& Dowben, P. A. (2020). The emergence of the local moment molecular spin transistor. Journal of Physics. Condensed Matter: An Institute of Physics Journal, 32(23), 234002. https://doi.org/10.1088/1361-648X/ab74e4 


\section{Introduction}

During the past few decades, "molecular spintronics" has been actively pursued because of the possibility of making nanoscale devices, utilizing the spin degree of freedom on the molecular scale [1-15]. This effort has been a combination of traditional molecular electronics with systems where the spin state matter, i.e. spintronics. Part of the motivation is the potential for addressing the complex grand challenge of manipulating magnetically ordered states by electrical means. The magnetic state may be switched through voltage control potentially without large current densities and power consumption, if the system is molecular, thus may lead to molecularly based nonvolatile device elements, and this has considerable appeal. To date, most efforts at providing magnetic memory element solid-state devices have used magnetic spin valves or magnetic tunnel junction types of structures. Many of the magnetic tunnel junction structures utilize inorganic dielectric thin films, combined with various magnetic layers, where large electrical current densities are required to reverse the direction of magnetization of one of the magnetic layers in the device structure [16-18]. This can be a big drawback.

Molecular systems have the very real possibility of providing a room temperature device on a length scale less than $10 \mathrm{~nm}$ while delivering low power nonvolatile local magneto-electric memory operations at peak (and only peak) current densities below $10^{4} \mathrm{~A} / \mathrm{cm}^{2}$, i.e. reduced power consumption (potentially to be less than $3 \mathrm{aJ}$ ). The nonvolatility that is possible with these local moment or spin crossover molecular systems may requires a structural change of some part of the molecular system or creation of a suitable heteromolecular systems. This latter route seems promising as cooperative effects have now been observed [19]. The intermolecular cooperative effects, if dominant, suggests that room temperature nonvolatile molecular devices are likely to require write times that are longer than a nanosecond or, possibly, much longer than a nanosecond. The molecular nature of such devices suggests that the devices themselves might be engineered to be flexible. Also of interest, but of lesser practical value, are devices where conductance and spin state are manipulated by large magnetic fields.

To achieve the electric control of the magnetic properties on the molecular scale, especially for a single molecule (or just a few molecules) in a device structure, molecules with a large local magnetic moment have attracted considerable attention. A large variety of organometallic and metal organic molecules exist, with one or more transition metal or rare earth metal ions as inner magnetic core and organic ligands surrounding the core as the shell [20,21], beginning with 
variants of $\left[\mathrm{Mn}_{12} \mathrm{O}_{12}(\mathrm{OAc})_{16}\left(\mathrm{H}_{2} \mathrm{O}\right)_{4}\right]$ [22-25]. The magnetic properties of these single molecule magnets (SMM) originate from the inner core, and often there exists a huge magnetic anisotropy within the molecule. The remanent magnetic moment, of the multiple metal atom core complexes, will depend on the alignment of the moments and, of course, applied field. The single ion single molecule molecular magnet frequently retain the magnetic anisotropy of the multiple metal atom core complexes, and the dysprosium metallocene cation complex $\left[\left(\mathrm{Cp}^{\mathrm{iPr} 5}\right) \mathrm{Dy}\left(\mathrm{Cp}^{*}\right)\right]^{+}\left(\mathrm{Cp}^{\mathrm{iPr} 5}\right.$, pentaiso-propylcyclopentadienyl; $\mathrm{Cp}^{*}$, pentamethylcyclopentadienyl) exhibiting something akin to magnetic hysteresis up to $80 \mathrm{~K}[26]$.

As molecules, the single molecule molecular magnets (SMM) have a highest occupied molecular orbital (HOMO) to lowest unoccupied molecular orbital gap, thus as a condensed molecular system have no free electron density, and there is no medium for exchange [27,28], while intermolecular dipolar coupling is weak because of the separation between the metal cores. Not surprisingly, few are ferromagnetic or even antiferromagnetic as the condensed solid state films. There is no Stoner-like exchange [29], but rather these are local moment systems in the condensed phase and the exchange splitting, on the molecular scale, is going to be insensitive to temperature. This is distinct from the spin crossover complexes [30,31], first noted as early as 1931 $[32,33]$, where the moment can vary from a low spin state, sometimes with zero net moment and thus exhibit diamagnetic behavior in the condensed solid state (as an ensemble of molecules) to a high spin state, thus showing moment paramagnetic behavior in the condensed solid state. In spite of the different between the single molecule molecular magnets with multiple metal atoms, the single ion single molecule molecular magnets, and the spin crossover complexes in the high spin state, all are local moment molecular systems.

Chemical modification provides a desirable way to tailor the organic ligand, which makes selective bonding to a particular surface possible [34], thus optimizing the stability of the device and potentially can provide a specific bonding orientation. One can also change the magnetic core and by doing so manipulate the magnetic properties of the molecule, without altering the structure significantly. Another way to switch the magnetization is through quantum tunneling. This phenomenon was observed in molecular crystals in 1996 [35,36]. When an external magnetic field is applied alone the easy axis of the molecular system, the degeneracy between $m_{s}$ and $-m_{s}$ is lifted (with positive $\mathrm{m}_{\mathrm{s}}$ being shifted up and negative shifted down), causing shifts in energy levels. 
In spite of the many complications faced, there has been progress towards making a threeterminal molecular spintronic device and the molecular spin transistor has, in fact, now been realized. There has been much interest especially in molecular spin transistors where only a single molecule is addressed in the device. As reviewed here, these devices tend to show functionality only at very low temperatures, but more recently, molecular thin film devices have been shown to exhibit isothermal conductance switching even at room temperature.

\section{Transport behavior of molecules in transistor geometry}

The single molecule transistor represents a large variety of three-terminal devices where a single molecule is embedded into a gap between the source and drain electrodes (Figure 1), and the gate adopted usually has the back-gated geometry, as reviewed elsewhere [27,28]. Two types of the gate materials have been typically chosen: highly doped silicon substrates with $\mathrm{SiO}_{2}$ grown on top or a native $\mathrm{Al}_{2} \mathrm{O}_{3}$ gate grown on top of aluminum. The choice of the gate material and thickness of the gate dielectric affects the break-down voltage of the gate oxide. The most challenging part is to fabricate nano-gaps between the source and drain electrodes, with a separation width of only one molecule. One commonly-used approach for creating such a junction is electromigration where breaking of the conducting channel material is induced by large current [27,28,37-40], to form the gap between the nominal source and drain. After the gap is formed by electromigration, molecules are introduced into the junction via solution by drop-casting. In principle, such gaps can also be created as break junction, the conducting wire is broken by mechanical strain.

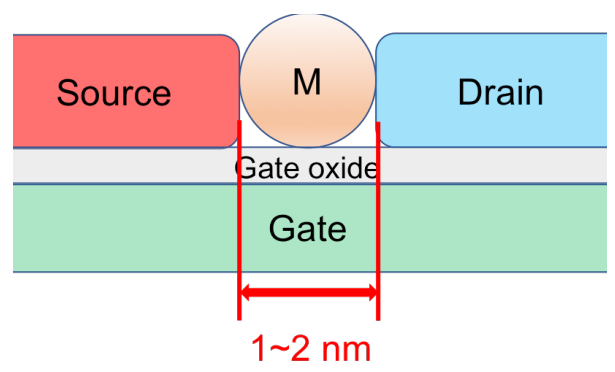

Figure 1. A schematic diagram of the single molecule transistor.

Once placed in the gap between source and drain of the transistor, single molecule magnets are not just the semiconductor channel of the transistor, but a quantum dot with discrete energy 
levels. When a molecule comes in contact with the metal leads (the source and drain electrodes), the metal will screen or perturb the molecular orbitals [41,42], causing a reduction of the HOMO (highest occupied molecular orbital) and LUMO (lowest unoccupied molecular orbital) gap. This molecular orbital perturbation may be considered as molecule-lead (electrode) coupling, represented by $\Gamma_{\mathrm{s}}$ and $\Gamma_{\mathrm{d}}$ (here "s" stands for source and "d" stands for drain) in Figure 2. These parameters affect tunneling rates and the overlap between the molecular wavefunction and the conduction electrons. The total coupling $\Gamma$, which is the sum of $\Gamma_{\mathrm{s}}$ and $\Gamma_{\mathrm{d}}$, is an indicator of the broadening of the molecular levels due to the hybridization between the molecule and leads. As $\Gamma$ gets bigger, the hybridization shifts both HOMO and LUMO closer to the Fermi Energy EF, i.e. the HOMO-LUMO gap closes as just noted, and the molecular levels broaden. Using electrochemical potential $\mu$, molecular states can be considered in terms of $\mu(\mathrm{N})$, defined as the total energy difference between the $\mathrm{N}$ electron ground state and N-1 electron ground state (Figure 2).

The tunneling transport in these molecular types of spintronic devices depends on the alignment between the chemical potentials of the molecule and leads (Figure 2). In experiments, the transport is commonly characterized by measuring the change of current I or differential conductance $\mathrm{d} / / \mathrm{dV}$ sd, which could be obtained by taking numerical differentiation of the I-V plot, versus the source-drain bias $V_{\text {sd }}$ under different gate voltages $V_{g}$ at very low temperature (usually in $\mathrm{mK}$ range). The figures plotted from these measurements are often called stability diagrams.
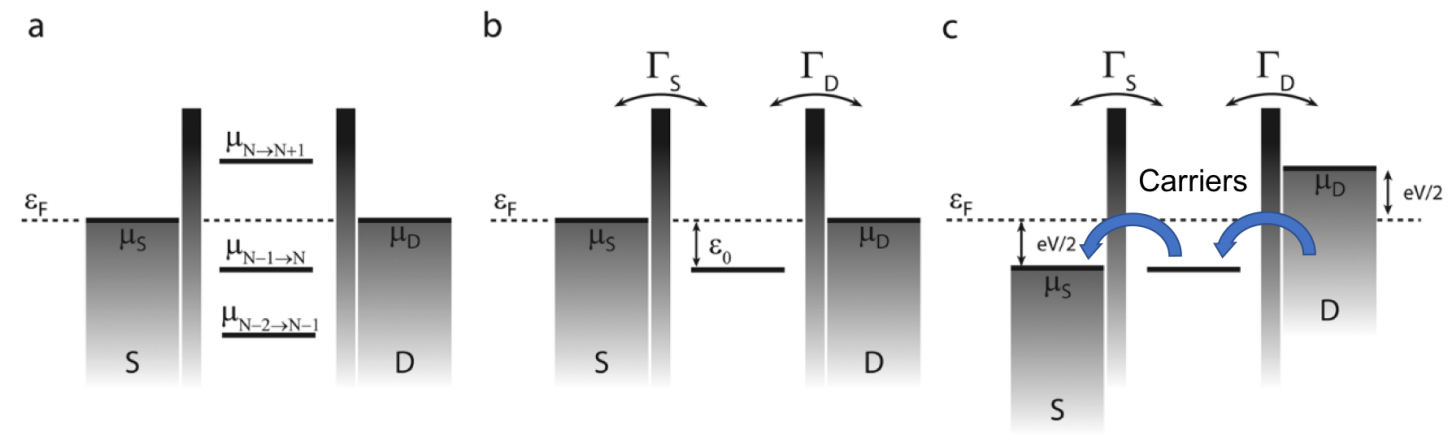

Figure 2. The chemical potential diagram of the molecular "channel" respect to the leads without applying gate voltage $(a, b)$ and under the influence of gate voltage V (c). Image reproduced from Ref. [27], with permission from The Royal Society of Chemistry. 
Unsurprisingly, a lot of the molecular spin transistors exhibit very low currents due to the single molecule nature of the device and thus have limited total conductance. Transport in single molecular transistors occurs via electron tunneling from the source to the molecule then tunnels out to the drain (co-tunneling). When the tunnel barrier resistance is much higher than the quantum of resistance $\left(\mathrm{h} / \mathrm{e}^{2}=25.8 \mathrm{k} \Omega\right)$, only one electron at a time can tunnel through the junction, and so places these devices in the framework of the single electron transistor [27]. While quantum conductance should go as $2 \mathrm{e}^{2} / \mathrm{h}$, where the factor 2 comes from electron spin degeneracy, this latter spin degeneracy is lifted when the molecule has a large magnetic moment.

When the reduction of the HOMO-LUMO gap is not big, i.e. in case of the weak coupling, between the local moment molecule and the electrodes, performing such experiments will show some interesting features: at low gate voltage $V_{g}$, there exists several regions of source-drain voltage, $\mathrm{V}_{\mathrm{sd}}$, and $\mathrm{V}_{\mathrm{g}}$ where there is no current (zero conductance) and this is referred to as Coulomb blockade. Coulomb blockade shows up as diamond shaped bounded regions (named Coulomb diamonds) on differential currents plots as a function of gate voltage $V_{g}$ and source-drain voltage, $\mathrm{V}_{\text {sd }}$ (Figure 3). Within each Coulomb diamond, the charge of the molecule is fixed to an integer number, and adjacent diamonds differ by only one charge (electron or hole).

Bright areas in the current plots (Figure 3b) denotes single electron tunneling region, where the transistor is in its "ON" state. The points connecting adjacent Coulomb diamonds are degeneracy points, which means at specific choices of $V_{s d}$ and $V_{g}$, the energy between two charge states, of the molecule, are the same. The gate voltage serves as a means to shift and "tune" the molecule chemical potential. When $\mathrm{V}_{\mathrm{sd}}=0, \mu_{\mathrm{s}}$ is aligned with $\mu_{\mathrm{d}}$. If the molecular level is shifted at the same chemical potential level as the leads (which is at the degeneracy point), electrons can tunnel through the molecule, resulting in a non-zero conductance signal, single electron tunneling could occur. When applying a non-zero voltage $\mathrm{V}_{\text {sd }}$, between the source and lead, the potential will shift the source chemical potential $\mu_{\mathrm{s}}$ up by $\frac{1}{2} e\left|V_{s d}\right|$ combined with pulling the drain $\mu_{\mathrm{d}}$ down by the same value, resulting in a bias window with a width of e $\left|\mathrm{V}_{\mathrm{sd}}\right|$ (Figure 2c). When the molecular level is correctly tuned within this bias window region, single electron transport will occur. 

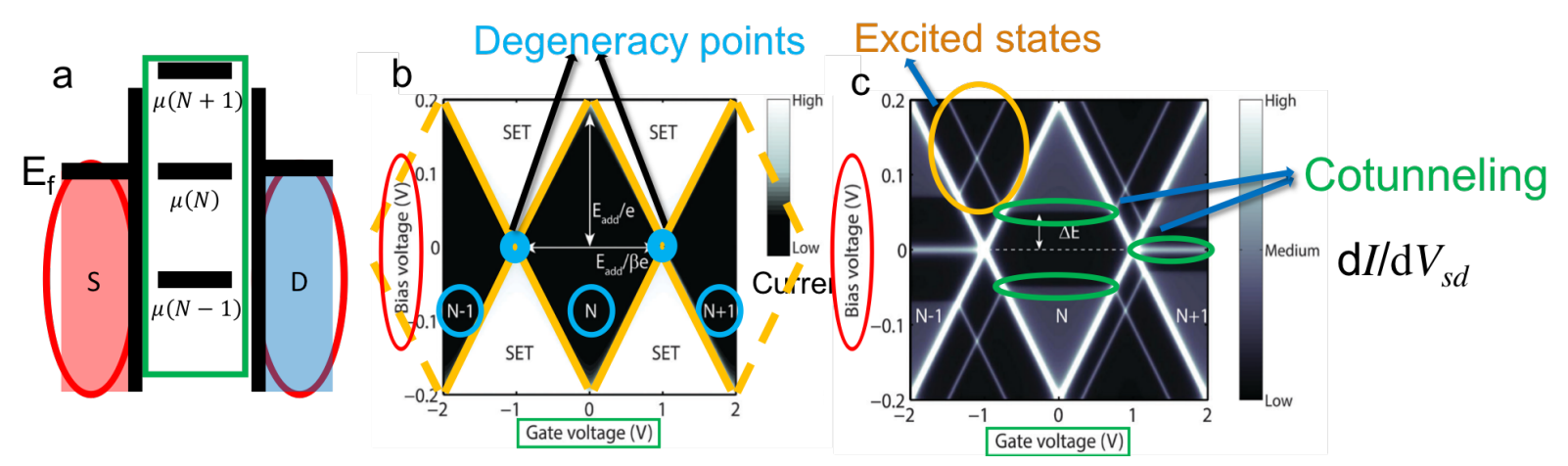

Figure 3. Schematic diagram of the current and differential conductance response for a single electron molecular transistor. (a) Chemical potential diagram of the molecule island (b) Current map versus bias voltage and gate voltage. (c) Differential conductance plotted versus bias voltage and gate voltage. Image(b,c) taken and reproduced from Ref. [27], with permission from the Royal Society of Chemistry.

The current, when plotted as differential conductance inside the single electron tunneling region, the parallel lines to the diamond edges (which could be seen in Figure 3c) are related to the contribution of the conductance due to excited states [27]. These excitations can have an origin related to charge, phonons (vibrational), and a variety of other optical excitations.

\subsection{Mn $\mathrm{Mn}_{12}$ family of local moment complexes}

Among the most studied single molecule magnets is the "Mn 12 " large spin moment class of molecules, discovered by Lis in 1980 [22], which contain a core of $12 \mathrm{Mn}$ atoms surrounded by acetate ligands. [ $\left.\mathrm{Mn}_{12} \mathrm{O}_{12}\left(\mathrm{O}_{2} \mathrm{C}-\mathrm{R}-\mathrm{SAc}\right)_{16}\left(\mathrm{H}_{2} \mathrm{O}\right)_{4}\right]$, or " $\mathrm{Mn}_{12}$ acetate" has, in fact, been used to make single molecule transistors. Absent ligand contributions, the molecule has a total spin $S=10$, with an Ising type anisotropy barrier of around $6 \mathrm{meV}[43,44]$. Heersche et al. [45] investigated the transport properties of two $\mathrm{Mn}_{12}$ derivatives $\left(\mathrm{Mn}_{12} \mathrm{O}_{12}\left(\mathrm{O}_{2} \mathrm{C}-\mathrm{R}-\mathrm{SAc}\right)_{16}\left(\mathrm{H}_{2} \mathrm{O}\right)_{4}\right]$ where $\mathrm{R}=\mathrm{C}_{6} \mathrm{H}_{4}$, $\mathrm{C}_{15} \mathrm{H}_{30}$ ), at a temperature of $3 \mathrm{~K}$, in 2006. In their device, they used thiol groups as the outer ligand to ensure a strong affinity to the gold surface so that the molecule is robustly tethered to the Au electrodes, while still weakly perturbed by the metallic contacts. Some interesting features appear in the differential conductance plots including regions with complete current suppression and negative differential conductance, as seen in Figure 4. At the degeneracy point, indicated on the left side of Figure 4a, the current is fully suppressed at some positive source-drain bias voltages (above the dashed line in Figure 4a) and restored at an excitation which appears $5 \mathrm{meV}$ higher. Two excitations at $2 \mathrm{meV}$ (positive differential conductance) and $3 \mathrm{meV}$ (negative differential 
conductance) are evident in the right side of Figure 4a. The current (I) versus source-drain bias plot shows this negative differential conductance feature as well (Figure 4b). Conventional
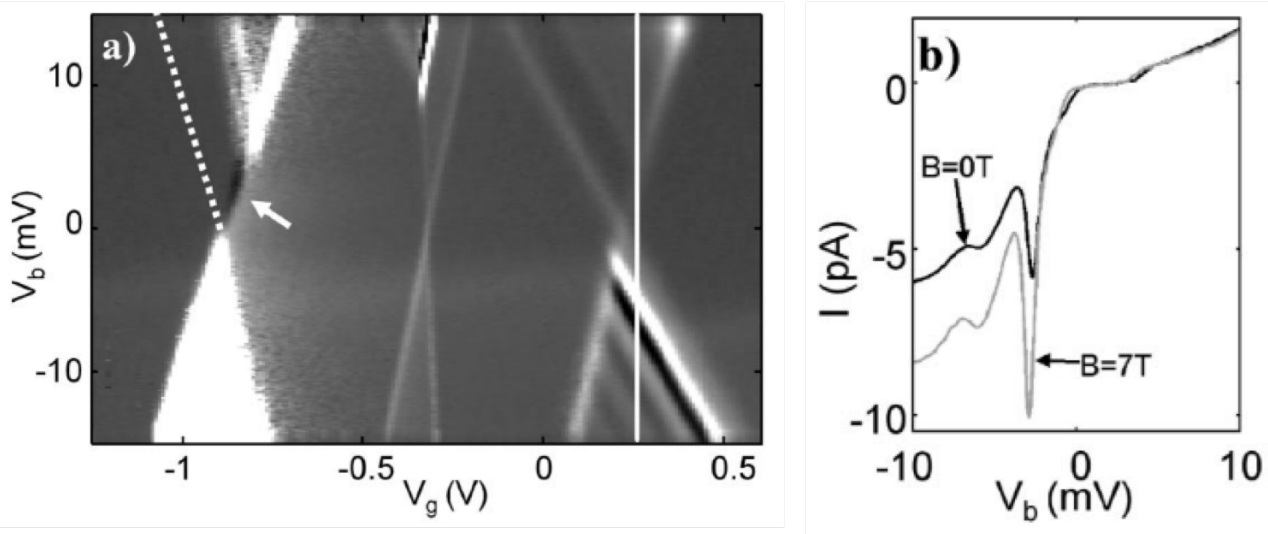

Figure 4. (a) Differential conductance stability diagram in gray scale of the $\mathrm{Mn}_{12}$ derivative $\left(\mathrm{Mn}_{12} \mathrm{O}_{12}\left(\mathrm{O}_{2} \mathrm{C}-\mathrm{R}-\mathrm{SAc}\right)_{16}\left(\mathrm{H}_{2} \mathrm{O}\right)_{4}\right]$, taken at a temperature of $3 \mathrm{~K}$. (b) the current versus voltage (I-V) curve, at the gate voltage indicated by white line at the left in panel (a). Image reprinted, with permission, from Ref. [45]. Copyright (2006) by the American Physical Society.

Coulomb blockade theory is an inadequate explanation [45], and this may be related to the charge induced distortion of the local spin state. In the context of their model, Heersche et al. [45] suggested that upon adding or subtracting one electron, the spin selection rule $|\Delta S|,\left|\Delta S_{z}\right|=1 / 2$ should apply for each energy allowed sequence. The subsequent sequential tunneling may result in the accumulation of a certain excited states while the relaxation of the moment could only occur by quantum tunneling of the magnetization, which is slow. Transport is thus hindered, and negative differential conductance and complete current suppression may occur [45]. Heersche et al. [45] also observed the enhancement of the current when an external magnetic field is applied to the device, but no specific conclusion was drawn. 

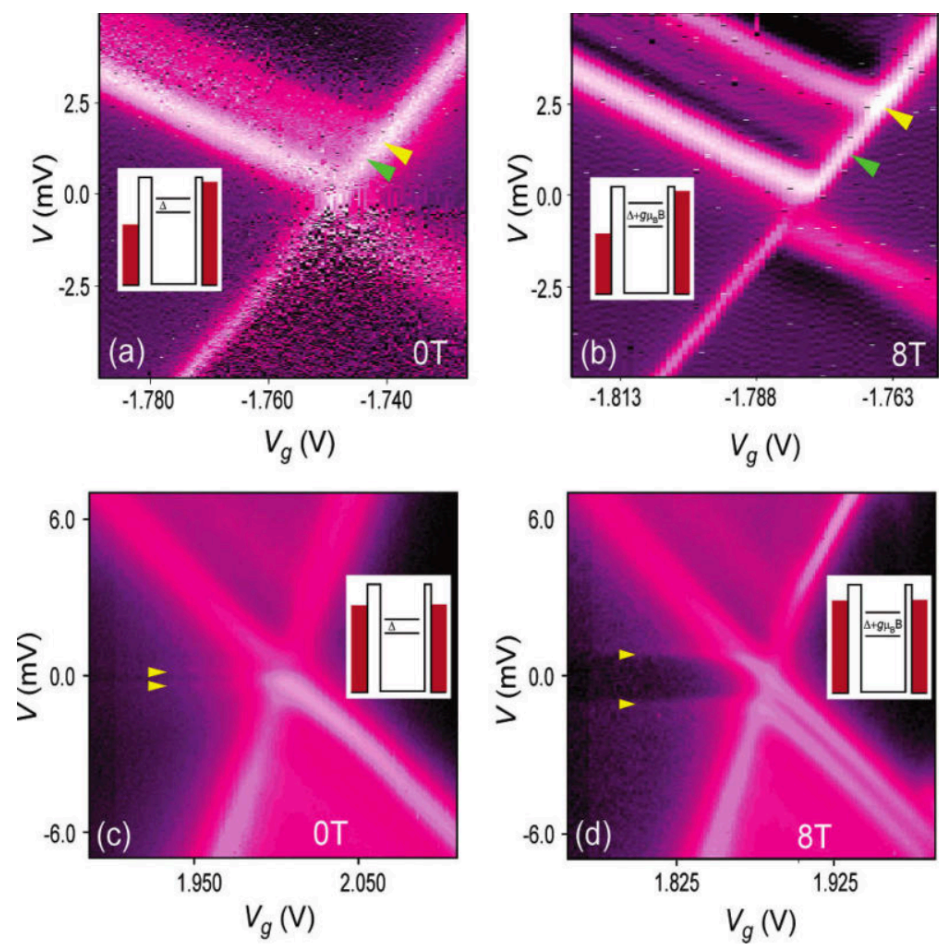

Figure 5. $(\mathrm{a}, \mathrm{b})$ The differential conductance stability plots for a " $\mathrm{Mn}_{12} \mathrm{Ac}$ " local moment molecular transistor, taken at $\mathrm{B}=0 \mathrm{~T}$ and $\mathrm{B}=8 \mathrm{~T}$. (c,d) The differential conductance stability plots for $\mathrm{Mn}_{12} \mathrm{Cl}$ local moment molecular transistor. The data was taken a temperature in the region $\leq 300 \mathrm{mK}$. Arrows indicating excited states. Image reprinted with permission from Ref. [46]. Copyright (2006), the American Chemical Society.

Later, Jo et al. [46] performed single molecule transistor measurements on $\mathrm{Mn}_{12} \mathrm{Ac}$ and $\mathrm{Mn}_{12} \mathrm{Cl}$ derivatives $\left(\mathrm{Mn}_{12}\right.$ derivatives $\left(\mathrm{Mn}_{12} \mathrm{O}_{12}\left(\mathrm{O}_{2} \mathrm{CCH}_{3}\right)_{16}\left(\mathrm{H}_{2} \mathrm{O}\right)_{4}\right.$, i.e. "MnAc" and $\mathrm{Mn}_{12} \mathrm{O}_{12}\left(\mathrm{O}_{2} \mathrm{CCHCl}_{2}\right)_{16}\left(\mathrm{H}_{2} \mathrm{O}\right)_{4}$, i.e. "MnCl"), mainly focusing on the magnetic field dependence at temperature less than $300 \mathrm{mK}$. For the transistor, based on $\mathrm{Mn}_{12} \mathrm{Ac}$ (Figure 5a,b), two excitations were observed at zero magnetic field B (yellow and green arrows). Upon applying a magnetic field of $8 \mathrm{~T}$, neither of the peaks (indicated by the yellow and green arrows in Figure 5a,b) have split (into the expected two peaks), meaning no simple Zeeman splitting occurs with this "MnAc" derivative [46], although the shift of one feature (indicated by the yellow arrow, Figure 5b) to higher energy is indicative of a Zeeman like effect. For $\mathrm{Mn}_{12} \mathrm{Cl}$ transistor (Figure 5c,d), Zeeman splitting is much more evident in the changes that occur in Coulomb blockade region of the differential conductance plot from Figure $5 \mathrm{c}$ to Figure $5 \mathrm{~d}$ (as indicated by the changing positions of the states indicated by the yellow arrows) [46]. 
The presence of a zero applied magnetic field splitting in the conductance plots, is certainly possible and is observed in the local moment of $\mathrm{Mn}_{12} \mathrm{O}_{12}\left(\mathrm{O}_{2} \mathrm{CCH}_{3}\right)_{16}\left(\mathrm{H}_{2} \mathrm{O}\right)_{4}$, i.e. "MnAc" system, as seen in Figure 6a [46]. A magnetic anisotropy barrier, Figure 6b [46], is challenged in the presence of an applied magnetic field as the splitting, in the conductance plots, decreases with increasing applied field. The steps in the plot of differential conductance, in Figure 6a may be related different available spin states, and the behavior overall is indicative of a magnetic anisotropy as discussed next.

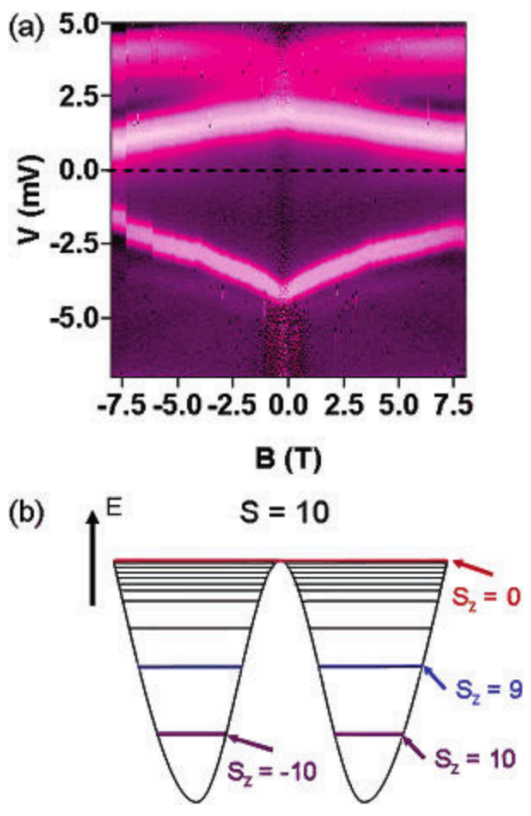

Figure 6. (a) The nonlinear evolution of the zero field split peaks (indicative of a large local moment magnetic system) as B sweeping for the molecular transistor based on the $\mathrm{Mn}_{12}$ derivatives $\left(\mathrm{Mn}_{12} \mathrm{O}_{12}\left(\mathrm{O}_{2} \mathrm{CCH}_{3}\right)_{16}\left(\mathrm{H}_{2} \mathrm{O}\right)_{4}, \mathrm{Mn}_{12} \mathrm{Ac}\right.$, taken at in the temperature region $\leq 300 \mathrm{mK}$, and (b) energy diagram of $\mathrm{S}=10$ state in $\mathrm{Mn}_{12}$ molecule. Image reprinted with permission from Ref. [46]. Copyright (2006), the American Chemical Society.

\section{The influence of magnetic anisotropy}

Molecular spin transistors using single molecule magnets has been studied in a large variety of molecules $[27,28,38,39]$ and device characteristics can be affected by more than just the spin and charge state. The magnetic property of a single molecule magnet might be roughly described as the following Hamiltonian (omitting thermal effects and higher order terms for simplicity) [27,28]:

$$
H=D S_{z}^{2}+E\left(S_{x}^{2}-S_{y}^{2}\right)+g \mu_{B} \mu_{0} \boldsymbol{S} \cdot \boldsymbol{H} \quad \text { eq. } 1
$$


Here $S_{x}, S_{y}$ and $S_{z}$ are spin operators in the three orthogonal directions, $D$ is the axial magnetic anisotropy while $E$ is the transverse magnetic anisotropy. Usually $|D|>>|E|$, resulting in a large magnetic anisotropy in z direction (easy axis along $S_{z}$ direction). The magnetic anisotropy means that single molecule magnets, with the ground state of spin $S$, can be divided into $(2 S+1)$ discrete energy values for different $\mathrm{m}_{\mathrm{s}}$ (from $-S$ to $S$ ) along $\mathrm{z}$ direction. The sign of the first term $D$ is negative, so the potential energy of the ground state forms a double well potential, so that states between $m_{s}$ and $-m_{s}$ is separated by an anisotropy barrier. The last term in Hamiltonian is associated with Zeeman energy, that occurs when an external magnetic field is applied to the system.

Due to the presence of the anisotropy barrier, in order to make a transition from $\mathrm{m}_{\mathrm{s}}$ to $-\mathrm{m}_{\mathrm{s}}$, i.e. to reverse the magnetization direction of the molecular local moment, one has to overcome the potential barrier with height $\left|D S_{z}^{2}\right|$, this could be done by the thermal activation or application of a sufficiently large magnetic field [46]. The relaxation time will increase in an exponential fashion as temperature goes down. For the $\mathrm{Mn}_{12} \mathrm{O}_{12}\left(\mathrm{O}_{2} \mathrm{C}-\mathrm{R}\right)_{16}\left(\mathrm{H}_{2} \mathrm{O}\right)_{4}$ class of $\mathrm{Mn}_{12}$ molecules, the local magnetization state is extremely long lived at temperatures in the region of $2 \mathrm{~K}$ [21].

\subsection{The $\mathrm{Fe}_{4}$ Molecular Complexes}

Three terminal local moment molecular spin devices, with a $\mathrm{Fe}_{4}$ metal core, have also been investigated (by Zyazin et al. [47] and Burzuri et al. [48]), much in the same was at the $\mathrm{Mn}_{12}$ molecular complexes just discussed. Three derivatives of the $\mathrm{Fe}_{4}$ metal core molecule $\left[\mathrm{Fe}_{4} \mathrm{~L}_{2}(\mathrm{dpm})_{6}\right](\mathrm{Hdpm}=$ 2,2,6,6-tetramethyl-heptan-3,5-dione) were studied with the ligand moieties $\mathrm{H}_{3} \mathrm{~L}=\mathrm{R}-\mathrm{C}\left(\mathrm{CH}_{2} \mathrm{OH}\right)_{5}$ with $\mathrm{R}=$ phenyl (i.e. " $\mathrm{Fe}_{4} \mathrm{Ph}^{\prime}$ ) and $\mathrm{R}=$ 9-(acetylsulfanyl)nonyl (i.e. " $\left.\mathrm{Fe}_{4} \mathrm{C}_{9} \mathrm{SAc}\right)$ [47], as well as $\left[\mathrm{Fe}_{4} \mathrm{~L}_{2}(\mathrm{dpm})_{6}\right] \cdot \mathrm{Et}_{2} \mathrm{O}\left(\mathrm{H}_{3} \mathrm{~L}=\right.$ 2-hydroxymethyl-2-phenylpropane1,3-diol) [48]. These complexes contains four $\mathrm{Fe}^{3+}$ ions, with one $\mathrm{Fe}^{3+}$ antiferromagnetically coupled to the other three and together the metal core is encapsulated in a hydrophobic shell made by ligands resulting in the total spin $\mathrm{S}=5$, as indicated in Figure 7, and magnetic anisotropy barrier of $16 \mathrm{~K}(1.378 \mathrm{meV})$ [47]. 


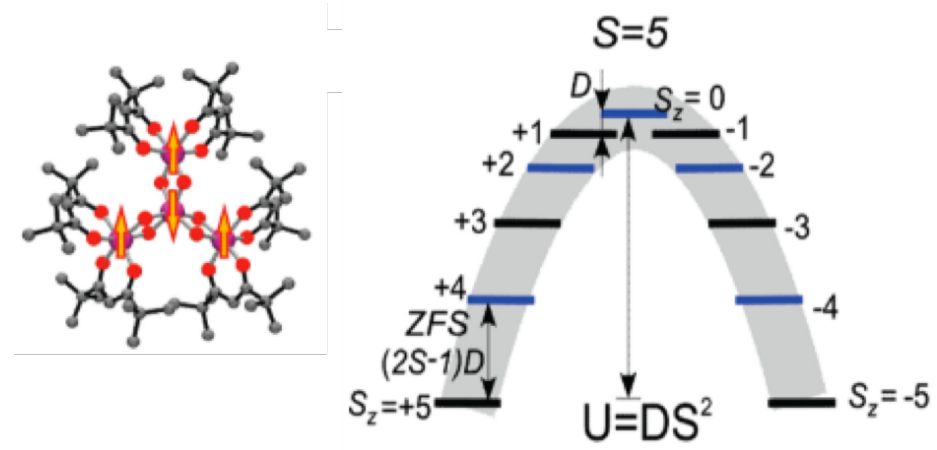

Figure 7. The generalized $\left[\mathrm{Fe}_{4} \mathrm{~L}_{2}(\mathrm{dpm})_{6}\right]$ molecule on the left and the spin state anisotropy diagram on the right, for such molecular systems with an $\mathrm{Fe}_{4}$ core, where the zero-field splitting, is denoted by ZFS. The image reprinted with permission from Ref. [47] with permission. Copyright (2010), the American Chemical Society.

With a single $\mathrm{Fe}_{4} \mathrm{Ph}$ or $\mathrm{Fe}_{4} \mathrm{C}_{9} \mathrm{SAc}$ complex, embedded between source and drain, in a gated three terminal device (like Figure 1), interesting transport properties were observed at $1.6 \mathrm{~K}$ [47]. Although the yield of successful devices was low [47,48], the signature of Coulomb blockade was observed [47,48], but so was inelastic cotunneling has been observed, with the data for the single $\mathrm{Fe}_{4} \mathrm{Ph}$ derivative shown in Figure 8a. The regions of single electron tunneling (SET) are also indicated.

The applied magnetic field dependence, shown in Figure 8b,c for the single $\mathrm{Fe}_{4} \mathrm{Ph}$ derivative, was related to the zero-field splitting of adjacent charge states (Figure 7). Within each Coulomb blockade area, the charge of the molecule was fixed, thus the magnetic anisotropy is fixed in the left and right region respectively of the differential conductance plot. The excitation energy of the left charge states is close to the zero-field splitting of the bulk crystal, assumed to be the neutral state with $|D|$ (of eq. 1) around $0.06 \mathrm{meV}$. Under the influence of an increasing applied magnetic field $\mathrm{B}$, the increase of the excitation energy is linear (Figure 8b,d), indicating a small angle between B and S [47]. Since a substantial angle will increase the transverse anisotropy component and can lead to a nonlinear behavior, which was observed for the $\mathrm{Fe}_{4} \mathrm{C}_{9} \mathrm{SAc}$ complex [47] and the $\mathrm{Fe}_{4}$ complex $\left[\mathrm{Fe}_{4} \mathrm{~L}_{2}(\mathrm{dpm})_{6}\right] \cdot \mathrm{Et}_{2} \mathrm{O}\left(\mathrm{H}_{3} \mathrm{~L}=\right.$ 2-hydroxymethyl-2-phenylpropane-1,3diol) [48]. The gate voltage will change the charge state of the molecule, changing its magnetic properties. Upon adding one electron into the molecule, the $\mathrm{B}$ dependence of the $\mathrm{N}+1$ charge state (Figure 8c,e) suggests a $|D|$ around $0.09 \mathrm{meV}$. 

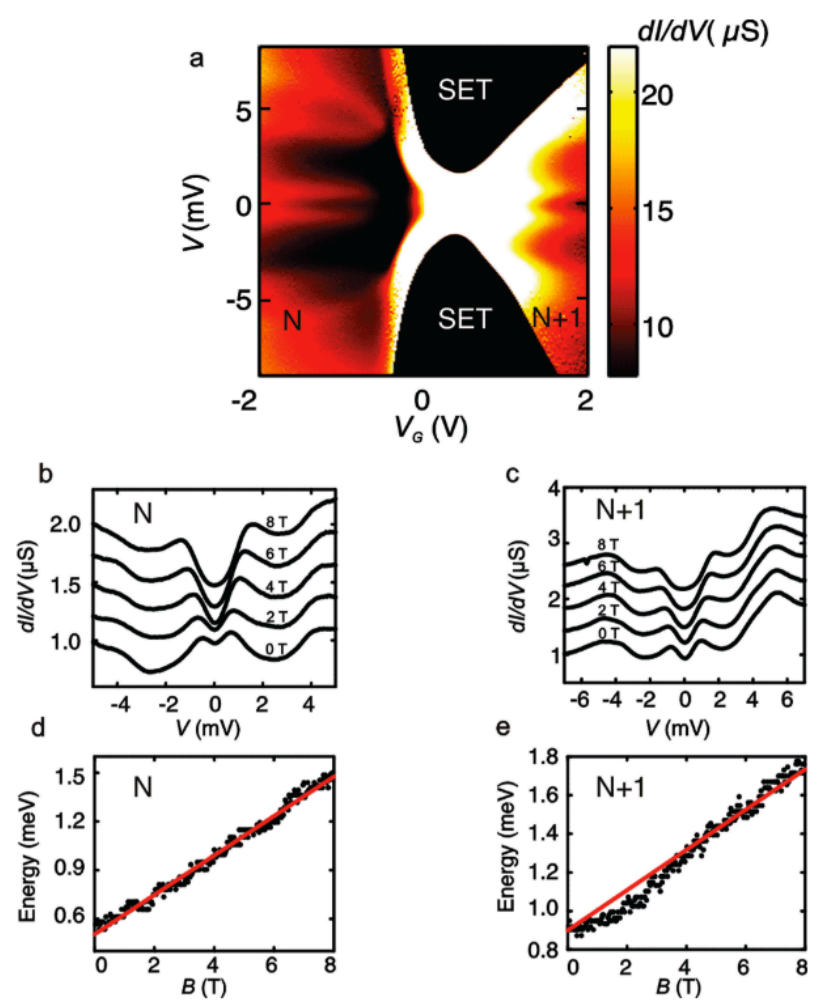

Figure 8. Differential conductance plots for the $\left[\mathrm{Fe}_{4} \mathrm{~L}_{2}(\mathrm{dpm})_{6}\right]$, i.e. an $\mathrm{Fe}_{4}$ complex, molecular spin transistor and B field dependency study, taken at 1.6 K. SET in (a) denoted the region of single electron tunneling. Image reprinted, with permission, from Ref. [47]. Copyright (2010) American Chemical

Society.

As indicated above, the evolution of the excitation energy, under applied magnetic field B, is related to the angle between $\mathbf{B}$ and $\mathbf{S}$. Burzurí et al. [48] performed an angular dependent magnetic field studies on the similar $\mathrm{Fe}_{4}$ complex $\left(\left[\mathrm{Fe}_{4} \mathrm{~L}_{2}(\mathrm{dpm})_{6}\right] \cdot \mathrm{Et}_{2} \mathrm{O}\right)$, where again Hdpm $=$ 2,2,6,6-tetramethyl-heptan-3,5-dione) and $\mathrm{H}_{3} \mathrm{~L}=$ 2-hydroxymethyl-2-phenylpropane-1,3-diol) spin transistors [48]. The changes in the energy levels, as a function of applied magnetic field, are clearly nonlinear in a zero bias Coulomb feature, in the differential conductance plots. The simulations (Figure 9b) suggest that the changes, with magnetic field, becomes more nonlinear as the angle between $\mathbf{B}$ and $\mathbf{S}$ increases, consistent with experiment (Figure 9c). The zero bias Coulomb feature, in the differential conductance plots, does show a deviation as the direction of the applied B field, relative to the device, has been rotated by 90 degrees, providing more direct proof of the magnetic anisotropy [48]. Kondo correlations were also observed [47,48], and we discuss Kondo effects next. 

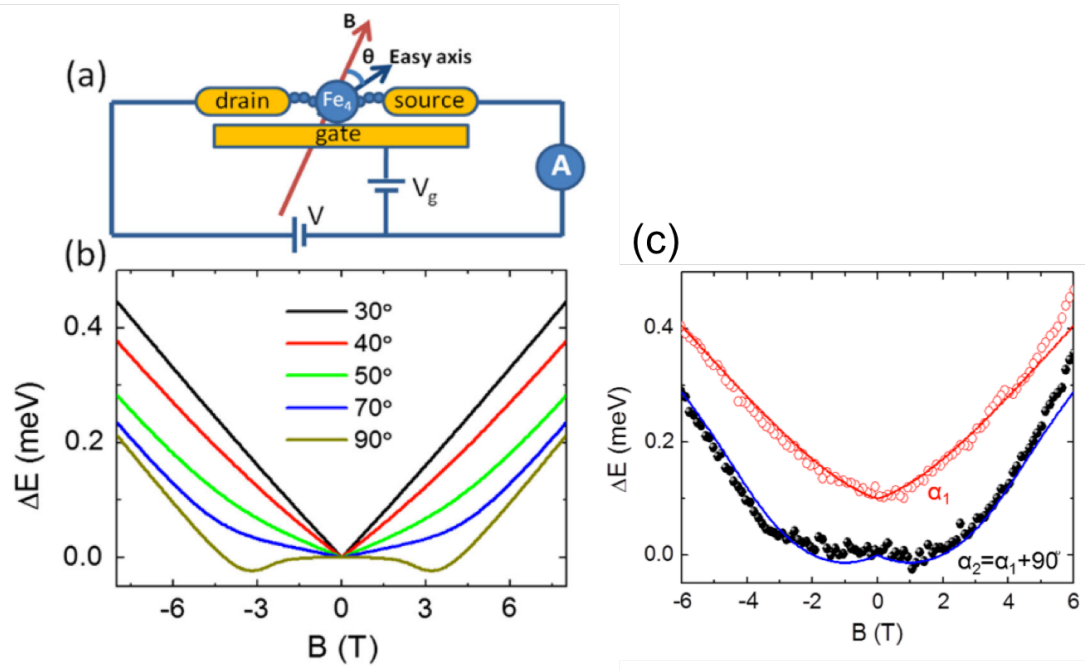

Figure 9. (a) The schematic of the molecular spin transistor, with the possible variation between the easy axis and applied magnetic field indicated, based on the $\mathrm{Fe}_{4}$ complex (complex $\left(\left[\mathrm{Fe}_{4} \mathrm{~L}_{2}(\mathrm{dpm})_{6}\right] \cdot \mathrm{Et}_{2} \mathrm{O}\right)$, where $\mathrm{H}_{3} \mathrm{~L}=$ 2-hydroxymethyl-2-phenylpropane-1,3-diol)). (b) The model of the shift in energy level, with applied magnetic field for various angles (only considering the $\mathrm{DS}_{\mathrm{z}}{ }^{2}$ and Zeeman term). (c) the experimental results of a zero bias Coulomb feature in the differential conductance plots, as a function of applied magnetic field $\mathbf{B}$ at two different angles, taken at $1.9 \mathrm{~K}$. Images reprinted, with permission, from Ref [48]. Copyright (2012) by the American Physical Society.

\section{Kondo effects in nominally single molecule transistor}

Higher order tunneling processes will show up when the coupling, i.e. interaction, between the molecule and the electrodes are enhanced [28,47-50]. In 2002, two papers [49,50], published together in Nature, highlighted Kondo effects in molecular spin transistors. Park et al. [49] made transistors with 2 similar Co based molecular complexes (Co bonded to two polypryridyl ligands, with thiol end groups, i.e. $\mathrm{Co}\left(\text { tpy- }\left(\mathrm{CH}_{2}\right)_{5}-\mathrm{SH}\right)_{2}$ and $\left.\mathrm{Co}(\text { tpy-SH})_{2}\right)$. One of the goals of this work was to change metal-organic complex coupling to the metal electrodes [49]. This change in the interaction of the Co(tpy) 2 molecular complex to the electrode, was affected by changing the length of the alkane thiol groups $\left(-\left(\mathrm{CH}_{2}\right)_{5}-\mathrm{SH}\right.$ versus $\left.-\mathrm{SH}\right)$ in ligands, as indicated in Figure 10a [49]. With the long alkane thiol moiety $\left(-\left(\mathrm{CH}_{2}\right)_{5}-\mathrm{SH}\right)$, the $\mathrm{Co}(\mathrm{tpy})_{2}$ molecular complex retained with behavior typical of a single electron transistor (Figure 10c), yet with a voltage control of the charge and spin state.

With the shorter alkane thiol moiety (-SH), the coupling of the Co(tpy) $)_{2}$ molecular complex has been enhanced and a zero-bias conductance Kondo resonance was observed (Figure 10d) [49], 
as was observed for $\left[\mathrm{Fe}_{4} \mathrm{~L}_{2}(\mathrm{dpm})_{6}\right]$ complexes discussed above [47,48]. Moreover, the Kondo resonance peak, for $\mathrm{Co}(\text { tpy-SH})_{2}$, splits in an applied magnetic field with spacing $2 \mathrm{~g} \mu_{\mathrm{B}} \mathrm{H}$, as seen in Figure 10d, [49]. The Kondo temperature was estimated to be around 10 25 K.
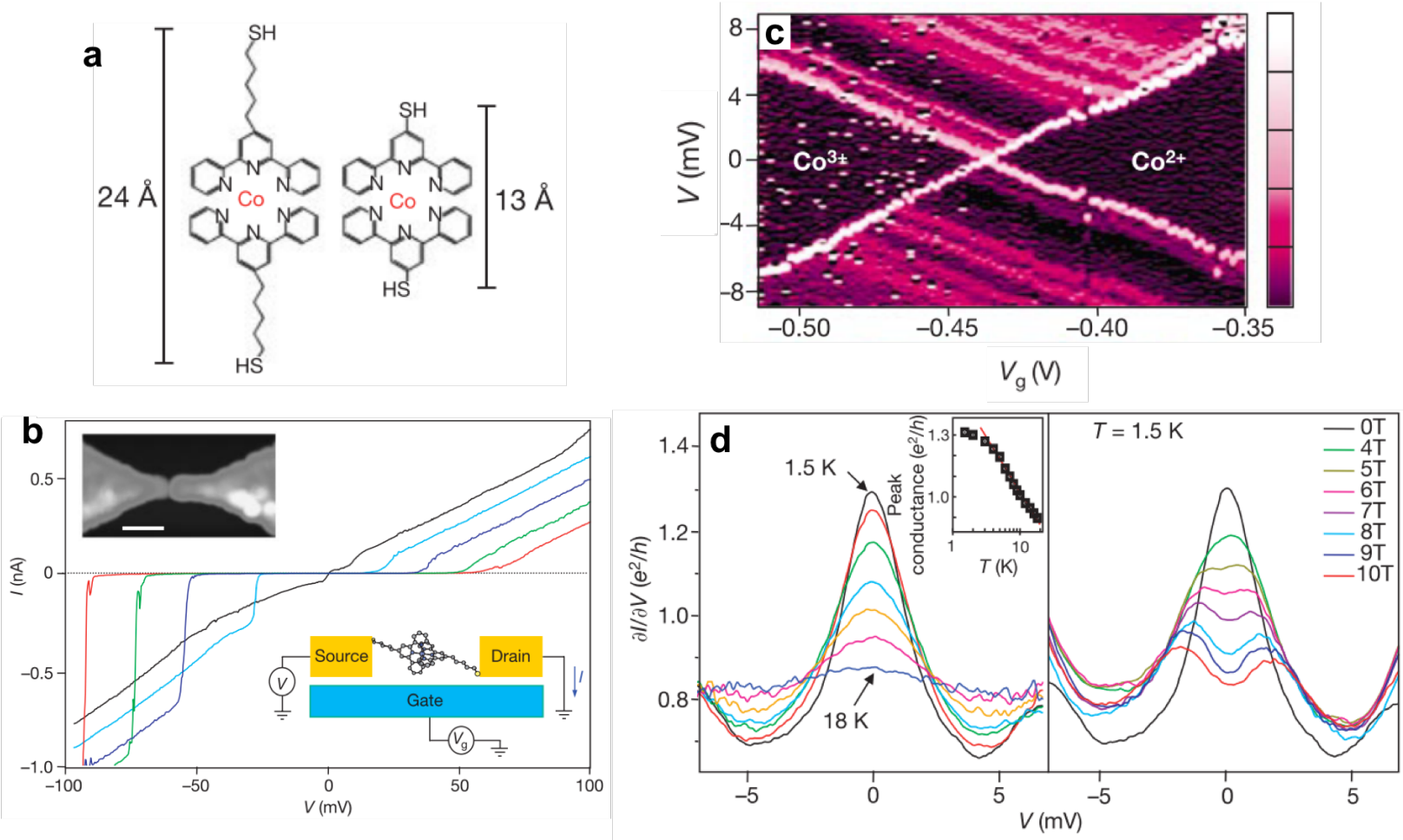

Figure 10. (a) The long and short Co complex molecules $\mathrm{Co}\left(\text { tpy- }\left(\mathrm{CH}_{2}\right)_{5}-\mathrm{SH}\right)_{2}$ and $\mathrm{Co}(\mathrm{tpy}-\mathrm{SH})_{2}$. (b) Device schematics and I-V curve showing the $\mathrm{Co}\left(\mathrm{tpy}-\left(\mathrm{CH}_{2}\right)_{5}-\mathrm{SH}\right)_{2}$ transistor conductance can be turned on and off. (c) the differential conductance diagram for $\mathrm{Co}\left(\operatorname{tpy}-\left(\mathrm{CH}_{2}\right)_{5}-\mathrm{SH}\right)_{2}$, with the long chain alkane thiol, as a function of source drain voltage (vertical) and gate voltage (horizontal), (d) the temperature evolution of the zero bias Kondo peak, on the left and the magnetic field dependence on the right, for Co(tpy-SH)2. Image adapted, by permission, from Ref. [49]: Springer Nature, Copyright (2002).

Liang et al. [50] investigated a single electron transistor fabricated with a divanadium $\left(\mathrm{V}_{2}\right)$ complex N, N', N"-trimethyl-1,4,7-triazacyclonane $)_{2}-\mathrm{V}_{2}(\mathrm{CN})_{4}\left(\mu-\mathrm{C}_{4} \mathrm{~N}_{4}\right)$, in a device much like that illustrated in Figure 1. The coupling is strong enough to observe a Kondo resonance within the left Coulomb diamond of the differential conductance plot (Figure 11a,b). Under the application of a magnetic field, the Kondo resonance undergoes Zeeman splitting, indicating a lifting of the spin 1/2 degeneracy at negative gate bias (Figure 11c,d). Upon adding one electron into the molecule, through an applied voltage, the spin degeneracy is lost and the Kondo resonance disappears [50]. 
The Kondo effect, for N, N', N"-trimethyl-1,4,7-triazacyclonane $)_{2}-\mathrm{V}_{2}(\mathrm{CN})_{4}\left(\mu-\mathrm{C}_{4} \mathrm{~N}_{4}\right)$, was retained up to $30 \mathrm{~K}$.

(a)

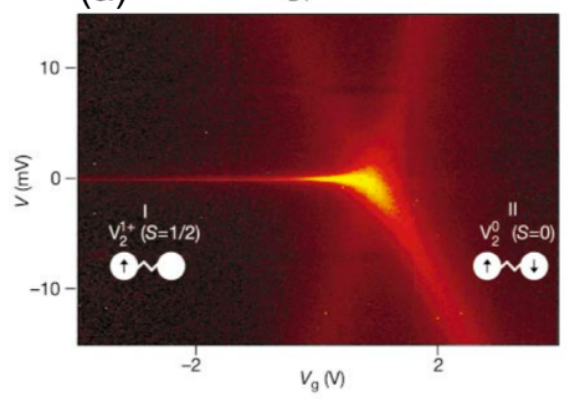

(b)

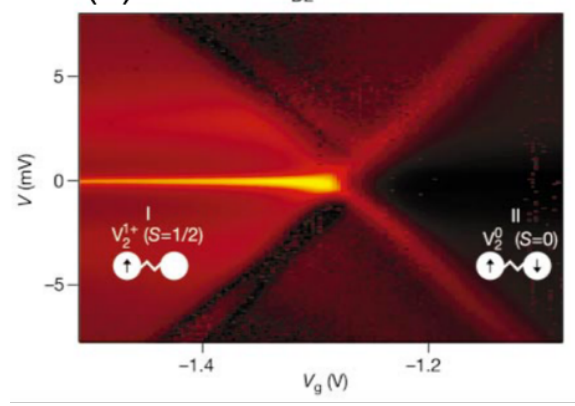

(c)

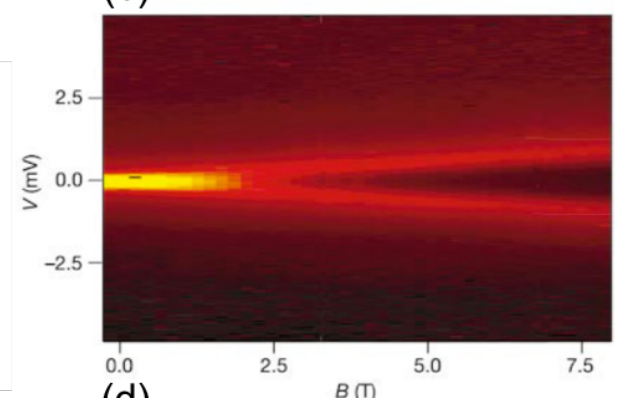

(d)

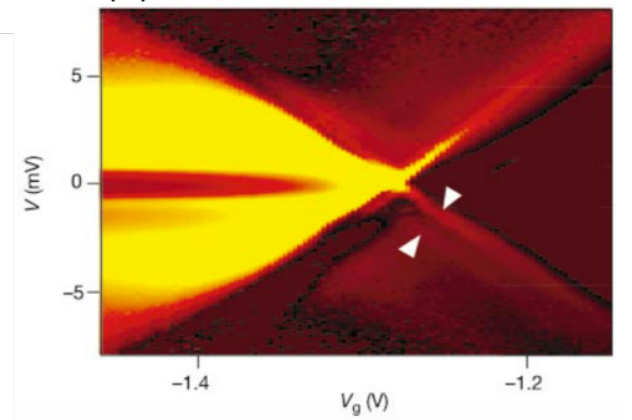

Figure 11. $(a, b)$ The differential conductance plots, for the $\mathrm{V}_{2}$ molecule $\left(\mathrm{N}, \mathrm{N}^{\prime}, \mathrm{N}\right.$ "-trimethyl-1,4,7triazacyclonane $\left.)_{2}-\mathrm{V}_{2}(\mathrm{CN})_{4}\left(\mu-\mathrm{C}_{4} \mathrm{~N}_{4}\right)\right)$, showing Kondo resonance in two individual devices (a) and (b). The inserted diagrams indicate the charge and spin state. (c) The Zeeman splitting under B field of the Kondo resonance peak and the differential conductance plot versus gate and source drain voltage, taken at an applied magnetic field of $8 \mathrm{~T}$. The data were acquired at $300 \mathrm{mK}$. Image adapted, by permission, from Ref [50]: Springer Nature, Copyright (2002).

Meded et al. [51] studied the electric control of an Fe(II) spin crossover complex $\left[\mathrm{Fe}^{\mathrm{II}}(\mathrm{bpp})_{2}\right]^{2+}(\mathrm{bpp}=2$, 6-bis(pyrazol-1-yl)pyradine $)$ in three-terminal geometry. Their DFT calculation showed that when the molecule charge falls to $q=+2$, the low spin state (LS) is more stable; for the charge neutral $q=0$, the high spin state (HS) becomes the ground state. Thus adding 2 electrons will trigger a transition from the LS state to the HS state. And the addition of electrons could be achieved by the gate voltage. Experimental results showed that upon adding electrons, the zero bias differential conductance changed, indicating a spin state transition (Figure 12). As temperature goes up, the variation of the conductance is reduced but as seen in Figure 12b, Kondo effects are evident [51]. The Kondo model fits, to the zero bias differential conductance data, 
suggests a Kondo temperature of $56 \mathrm{~K}$ for this $\left[\mathrm{Fe}^{\mathrm{II}}(\mathrm{bpp})_{2}\right]^{2+}(\mathrm{bpp}=2$, 6-bis(pyrazol-1-yl)pyradine) device [51].
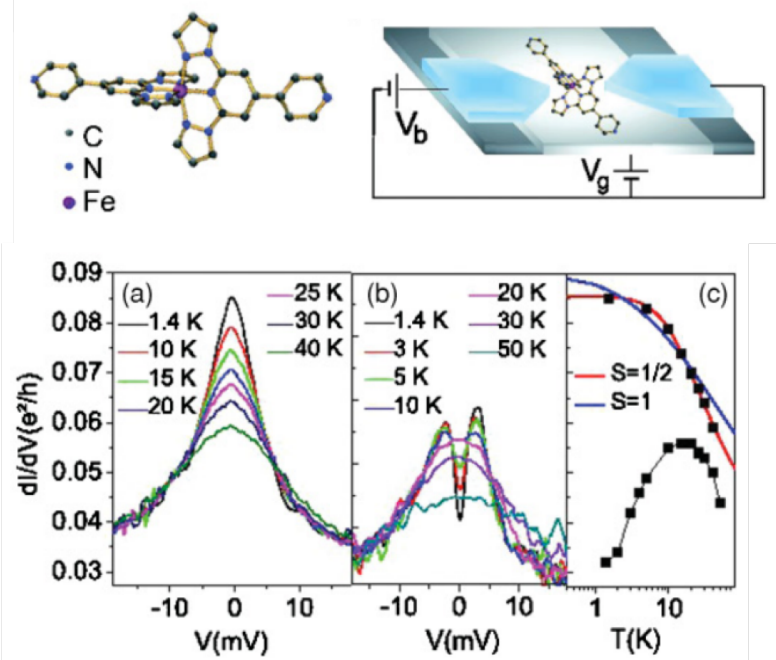

Figure 12. Top: the spin crossover complex molecule and device geometry for the single $\left[\mathrm{FeII}(\mathrm{bpp})_{2}\right]^{2+}$ molecular spin crossover channel transistor. $(a, b)$ Temperature dependence of differential conductance versus bias voltage, near zero bias, showing the temperature dependence of the Kondo peak. (a) is data taken for a gate voltage of $2.2 \mathrm{~V}$, (b) is taken for a gate voltage $3.2 \mathrm{~V}$. (b) Similar measurement when 2 electrons are introduced. (c) Temperature dependence of the zero-bias conductance in $(a, b)$. Image adapted, with permission, from Ref [51]. Copyright (2011) by the American Physical Society.

\section{Probing the nuclear spin}

The single molecule magnet $\mathrm{TbPc}_{2}$, where a single $\mathrm{Tb}^{3+}$ ion is sandwiched by two organic phthalocyanine (Pc) ligands, has also attracted attention [52-56]. Part of the appeals is that this metal-phthalocyanine molecule has a magnetic ground state of $\mathrm{J}=6$ with very strong uniaxial magnetic anisotropy, along the normal to the phthalocyanine plane (as indicated in Figure 12a), due to the strong spin-orbit coupling of the complex. When connected to an electric bias, current flows more favorably through the $\pi$ conjugated Pc ligands [52]. Because of the huge energy gap from the $J_{z}= \pm 6$ ground state to the first excited state (corresponding to a few hundred Kelvin), the molecule will stay in a stable ground state when experiments are performed at lower temperatures $(<5 \mathrm{~K})$ and small magnetic fields $(<10 \mathrm{~T})$. Due to the hyperfine coupling between the nuclear spin $\mathrm{I}=3 / 2$ and $\mathrm{Tb}^{3+}$ local moment, due to unpaired electron spins, the ground state $\mathrm{J}_{\mathrm{z}}=+6$ of this molecule will split into hyperfine 4 states with $\left|J_{z}, I_{z}>=\right|+6,+3 / 2>,|+6,+1 / 2>|+6,,-1 / 2>$, and $\mid+6$, 
$-3 / 2>$, and do so similarly for $J_{Z}=-6$, as indicated in Figure $12 b$. At small applied magnetic fields, a transition could occur, for example between $\mid+6,+1 / 2>$ and $\mid-6,+1 / 2>$ via quantum tunneling, at the avoided energy level crossings, while at higher magnetic fields getting larger, direct relaxation are possible, leading to the reversal of $\mathrm{J}$ (Figure 13b) [52].

Vincent et al. [39] explored $\mathrm{TbPc}_{2}$ in a three-terminal molecular transistor and achieved the probing of the nuclear spin via electrical means and a direct proof of quantum tunneling of magnetization (QTM). Sweeping the magnetic field near the degeneracy point, abrupt jumps of differential conductance, $d I / d V$, were observed showed up at 4 discrete values of magnetic field $\mathrm{B}$ (Figure 14b), associated with the reversal of $\mathrm{Tb}^{3+}$ magnetic moments, resulting from quantum tunneling of magnetization [53]. Statistical studies verified the precision of these applied magnetic field values quantum tunneling changes of magnetization (Figure 13c). Later, Thiele et al. utilized this read-out mechanism and achieved some control of the hyperfine interactions using an electric field pulse [53]. Other differential conductance change experiments for $\mathrm{TbPc}_{2}$ [54], provided evidence of the direct reversal of magnetization, at higher magnetic fields, rather than quantum tunneling as well as evidence for electron phonon coupling.
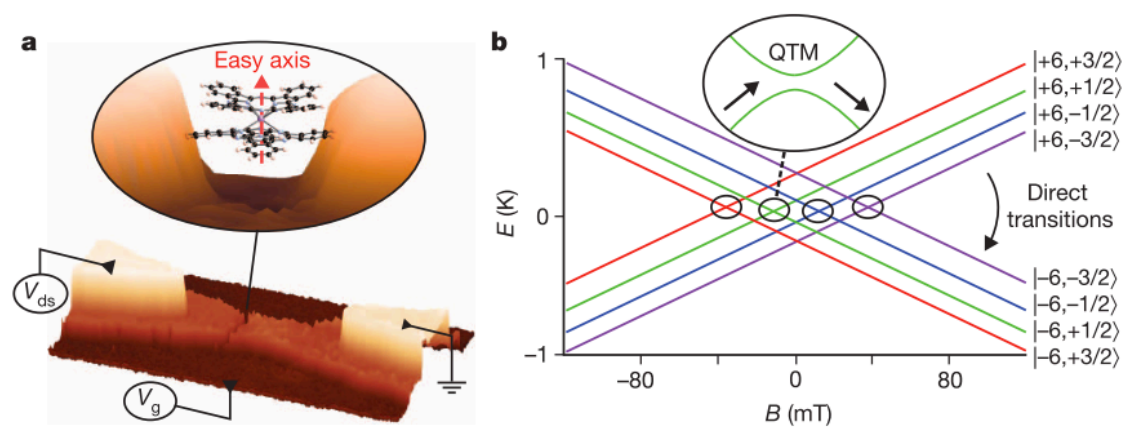

Figure 13. (a) The $\mathrm{TbPc}_{2}$ molecular spin transistor and (b) the energy diagram of spin reversal processes. In (b), two different mechanisms (quantum tunneling and direct reversal) are indicated. Image reprinted by permission from Ref [52]: Springer Nature, Copyright (2012). 

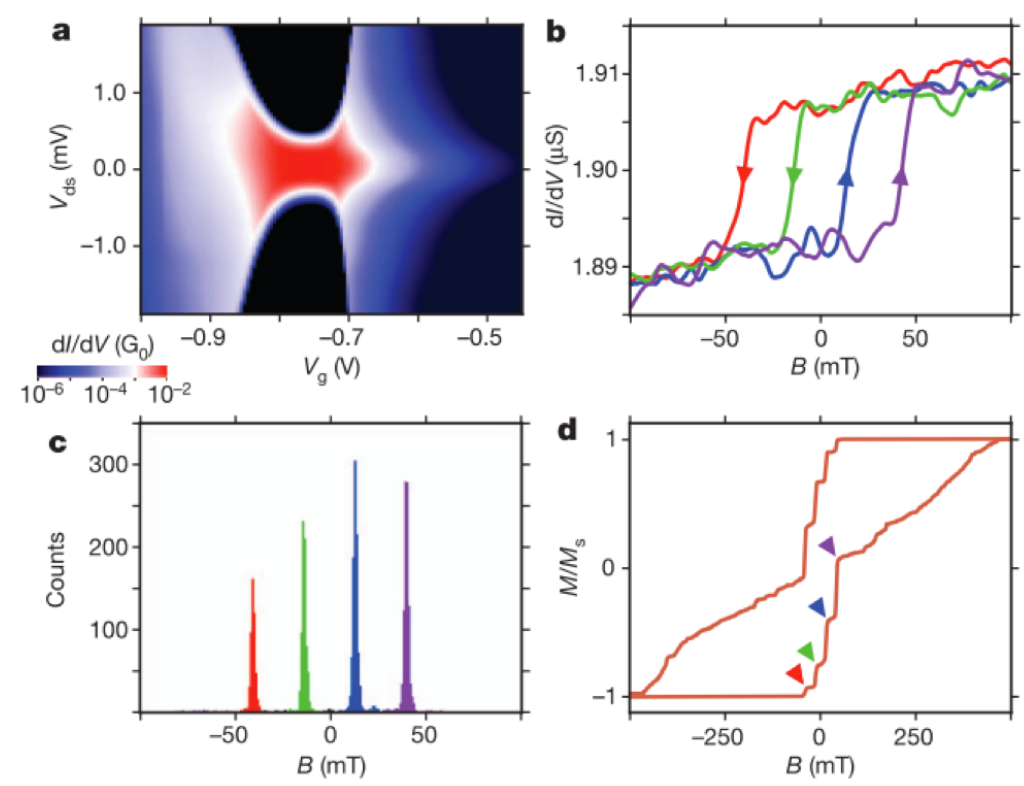

Figure 14. The conductance characteristics of $\mathrm{TbPc}_{2}$ transistor at temperatures less than $5 \mathrm{~K}$. (a) the differential conductance plot versus gate and source-drain voltage (b) the applied magnetic field B dependence for a gate voltage of $\mathrm{Vg}=-0.9 \mathrm{~V}$ and source drain voltage of $\mathrm{V}_{\mathrm{sd}}=0 \mathrm{~V}$, where the arrows indicate the direction of magnetic field sweep. The abrupt jumps were associated with quantum tunneling of magnetization (QTM). (c) Statistical results from 11000 trials sweeping the magnetic field, showing the robustly discrete quantum tunneling of magnetization (QTM). (d) Hysteresis generated by integration of 1000 trials, with the arrows indicating the 4 discrete magnetic fields associated with QTM events (-40 $\mathrm{mT}$ (red), -14 mT (green), $14 \mathrm{mT}$ (blue) and $40 \mathrm{mT}$ (purple)). Image adapted by permission from Ref

[52]: Springer Nature, Copyright (2012).

We suggest that equivalent complex, with praeseodynium instead of terbium, very might be interesting for comparison, as ferromagnetism in Pr arises from the nuclear moment, through the hyperfine interaction, at extremely low temperatures.

\section{Molecular spin transistor using spin crossover molecular thin films}

The molecular transistors, discussed so far, have not been accompanied by any evidence of nonvolatile switching at room temperature: the device measurement were carried out at temperatures below $30 \mathrm{~K}$. To obtain molecular spintronic devices functional, operational at temperatures closer to room temperature route, the molecular systems exhibiting a robust spin transition have begun to attract increasing attention [1-15]. The spin crossover phenomenon, 
representing transitions between two distinguishable spin states, occurs in a large variety of $3 d$ transition metal molecular compounds [30,31]. The two electronically distinct spin states, usually called low spin state (LS) and high spin state (HS), can be reversibly switched. Various external stimuli can trigger this spin transition, such as temperature, pressure, light, but above all electric field $[7,8,15,39,44,45]$. For practical point of view, if spin crossover molecules are expected to be used for solid state devices, there must be a way to "easily" sense the change of the spin state so that the state could be "read", and among the most desirable read mechanisms would be a conductance change, as seen in the experiments discussed above. The spin transition is seen to be accompanied by a conductance change $[4,5,7,8,12-14,15,52,57,58]$, as reviewed by Lefter et al. [15]. Furthermore, isothermal changes in the electronic structure and spin state have now been achieved for the $\mathrm{Fe}$ (II) spin crossover complex $\left[\mathrm{Fe}\left\{\mathrm{H}_{2} \mathrm{~B}(\mathrm{pz})_{2}\right\}_{2}\right.$ (bipy)] [5,9], $\left[\mathrm{Fe}(\operatorname{trz})_{3}\right]\left(\mathrm{BF}_{4}\right)_{2}(\operatorname{trz}$ $=$ triazole $)][14],\left[\mathrm{Fe}(\text { phen })_{2}(\mathrm{NCS})_{2}\right][7]$ and $\left\{\mathrm{Rb}_{0.8} \mathrm{Mn}\left[\mathrm{Fe}(\mathrm{CN})_{6}\right]_{0.93} \cdot 1.62 \mathrm{H}_{2} \mathrm{O}\right\}$ [12] at or near room temperature. More recently, magnetoelectric effects have been observed for $\mathrm{NiCl}_{2}-$ $4 \mathrm{SC}\left(\mathrm{NH}_{2}\right)_{2}$ [59], $\mathrm{CuCl}_{2} \cdot 2\left[\left(\mathrm{CH}_{3}\right)_{2} \mathrm{SO}\right]$ [60] and $\left[\mathrm{Mn}^{3+}(\text { pyrol })_{3}(\right.$ tren)] [61], which certainly has device implications [62], if this can be demonstrated reliably at room temperature.

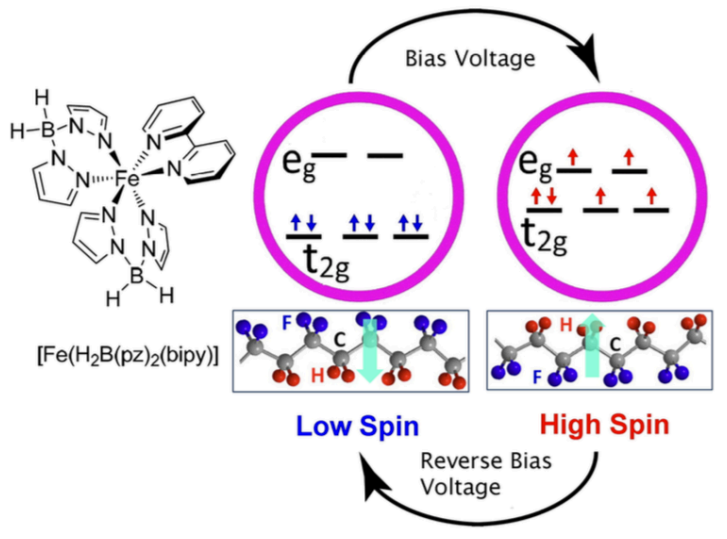

Figure 15. Schematics of voltage control of spin state switching by ferroelectric substrate polarization change. Image reprinted from Ref. [57], with the permission of AIP Publishing.

Zhang et al. [63] showed that for spin crossover molecular films of [ $\mathrm{Fe}\left(\mathrm{H}_{2} \mathrm{~B}(\mathrm{pz})_{2}\right)_{2}$ bipy], where $\mathrm{pz}=\operatorname{tris}\left(\right.$ pyrazol-1-yl)-borohydride and bipy $=2,2^{\prime}$-bipyridine, on top of organic ferroelectric substrate PVDF-TrFE, the direction of the interface dipole moment will lock the spin state of $\left[\mathrm{Fe}\left(\mathrm{H}_{2} \mathrm{~B}(\mathrm{pz})_{2}\right)_{2}\right.$ bipy] into either the high spin or low spin configuration depending on the direction of the moment, as schematically illustrated in Figure 15, and more recently repeated on 
the molecular ferroelectric polyvinylidene fluoride hexafluoropropylene (PVDF-HFP) [58]. This result was explored further to demonstrate isothermal reversible spin state switching, at room temperature, through an applied voltage [58]. Molecular thin films of the spin crossover complex $\left[\mathrm{Fe}\left(\mathrm{H}_{2} \mathrm{~B}(\mathrm{pz})_{2}\right)_{2}\right.$ bipy] grown adjacent to the organic ferroelectrics PVDF-HF and croconic acid, were seen to have nonvolatile locking of the spin state, but the spin state could be then manipulated by an applied electric field [58]. The spin state was directly probed by X-ray absorption spectroscopy, but the spin state switching was seen to be accompanied by a conductance change [58]. The high spin state was seen to have higher conductance than the low spin state (Figure 16). There is now at least one example of voltage controlled nonvolatile reversible spin state switching at room temperature.

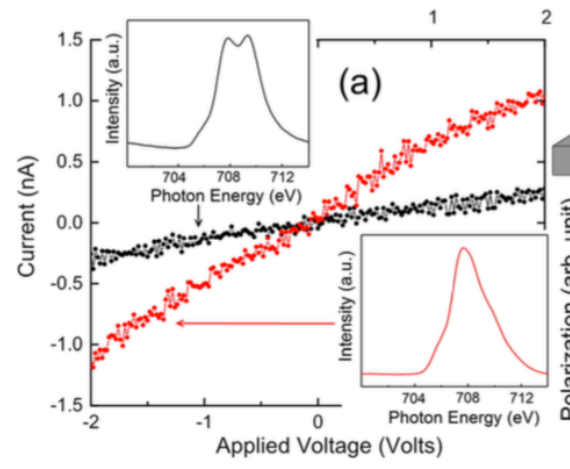

(b)

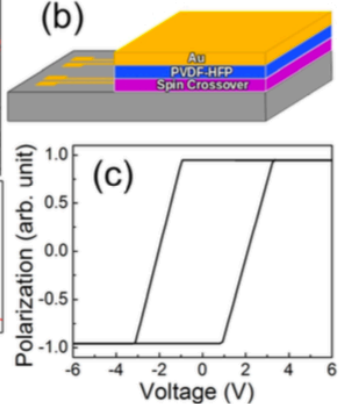

Figure 16. (a) The transport measurement and indications of conductance change associated with the spin state switching. The insets are the XAS spectra across the Fe $2 p_{3 / 2}$ core level indicating a change from the mostly low spin state to the high spin state for the $\left[\mathrm{Fe}\left(\mathrm{H}_{2} \mathrm{~B}(\mathrm{pz})_{2}\right)_{2}\right.$ bipy] on the organic ferroelectric polyvinylidene fluoride hexafluoropropylene (PVDF-HFP) polarized towards the $\left[\mathrm{Fe}\left(\mathrm{H}_{2} \mathrm{~B}(\mathrm{pz})_{2}\right)_{2}\right.$ bipy] (red) or away (black). (b) The schematics of the transistor device. (c) The hysteresis loop of the ferroelectric substrate. Image reprinted from Ref. [57], with the permission of AIP Publishing.

\section{Conclusion}

Obviously, there is now considerable evidence that gated molecular spin devices, using single (or only a few) molecule magnets, can be fabricated. First, although electromigration and break-junction devices technique have been widely used for the fabrication of single molecule devices, the success rate still remains low. It appears that only a small fraction of the devices fabricated showed the desired single electron transport characteristics such as Coulomb blockade 
and Kondo effects [45-51]. Nor is it easy to establish beyond any shadow of doubt whether the single molecule devices are indeed restricted to a single molecule, as opposed to few-molecule, devices. For molecules coupled, i.e. in electrical contact, to the metal, there might be a size mismatch so fine tuning the devices source-drain gap, to fit the molecule of interest, and then inserting the molecule correctly may be regarded as impressive technical challenges. Avoiding screening, by the high electron density of the adjacent metal electrodes can be done by chemical means, as indicated above, but by no means can one completely avoid a closing of the HOMO LUMO gap due to proximity of the adjacent metal. Using organic electrodes such as graphene or carbon nanotubes may solve this problem and broaden the choices of ligand groups, while possibly providing a route to more controlled if not more facile charge injection. Several groups have begun utilizing this idea and some progress has been made [55,64]. Furthermore, so far there is no way to precisely control the orientation of the molecule, when embedding a local moment molecule in the region between the source/drain electrodes, and how this influences the easy axis of the molecular magnetic anisotropy still remains unclear.

Another big issue to address is that all the single molecule devices reviewed here work only at very low temperatures (far below $40 \mathrm{~K}$ ). Electrical tuning of the molecular spin states using gate voltage is of great importance, in molecular spintronics, but to be a "true" device applicable to memory applications, this has to work at room temperature. The grand challenge is to achieve the electric tuning of the molecular spin states in a nonvolatile and reversible way around room temperature, so that better low power consumption spintronic devices could be designed and utilized. The specific challenges include 1) the ability to make a thin film, 2) the ability to "lock" the spin state, i.e. achieve nonvolatility, 3) the ability to isothermally "unlock" and switch the spin state, ideally with voltage, 4) obtain a usable readout mechanism, like a conductance change with spin state, which then needs to be accompanied by 5) high on/off ratios, 6) room temperature operation, 7) reasonably fast read and write operations (ideally $1 \mathrm{~ns}$ or less), and 8) sufficiently robust to survive $10^{16}$ switches. As yet, not all these conditions have been met in a working molecular spintronics device. Yet there is in fact considerable progress in meeting the challenges that need to be met to build scalable molecular spintronic memory. 


\section{Acknowledgements}

This research was supported by the National Science Foundation through NSF-Chem 1856614. The authors have benefitted from numerous discussions with Prof. Xiaoshan Xu. 


\section{Reference}

[1] Cornia A and Seneor P 2017 The molecular way Nat. Mater. 16 505-6

[2] Ludwig E, Naggert H, Kalläne M, Rohlf S, Kröger E, Bannwarth A, Quer A, Rossnagel K, Kipp L and Tuczek F 2014 Iron(II) Spin-Crossover Complexes in Ultrathin Films: Electronic Structure and Spin-State Switching by Visible and Vacuum-UV Light Angew. Chemie Int. Ed. 53 3019-23

[3] Rotaru A, Dugay J, Tan R P, Guralskiy I A, Salmon L, Demont P, Carrey J, Molnár G, Respaud M and Bousseksou A 2013 Nano-electromanipulation of Spin Crossover Nanorods: Towards Switchable Nanoelectronic Devices Adv. Mater. 25 1745-9

[4] Rotaru A, Gural'skiy I A, Molnár G, Salmon L, Demont P and Bousseksou A 2012 Spin state dependence of electrical conductivity of spin crossover materials Chem. Commun. 48 $4163-5$

[5] Ruiz E 2014 Charge transport properties of spin crossover systems Phys. Chem. Chem. Phys. 16 14-22

[6] Kuch W and Bernien M 2017 Controlling the magnetism of adsorbed metal-organic molecules J. Phys. Condens. Matter 29023001

[7] Miyamachi T, Gruber M, Davesne V, Bowen M, Boukari S, Joly L, Scheurer F, Rogez G, Yamada T K, Ohresser P, Beaurepaire E and Wulfhekel W 2012 Robust spin crossover and memristance across a single molecule Nat. Commun. 3938

[8] Gopakumar T G, Matino F, Naggert H, Bannwarth A, Tuczek F and Berndt R 2012 Electron-Induced Spin Crossover of Single Molecules in a Bilayer on Gold Angew. Chemie Int. Ed. 51 6262-6

[9] Zhang X, Costa P S, Hooper J, Miller D P, N'Diaye A T, Beniwal S, Jiang X, Yin Y, Rosa P, Routaboul L, Gonidec M, Poggini L, Braunstein P, Doudin B, Xu X, Enders A, Zurek E and Dowben P A 2017 Locking and Unlocking the Molecular Spin Crossover Transition Adv. Mater. 291702257

[10] Molnár G, Rat S, Salmon L, Nicolazzi W and Bousseksou A 2018 Spin Crossover Nanomaterials: From Fundamental Concepts to Devices Adv. Mater. 301703862

[11] Pronschinske A, Chen Y, Lewis G F, Shultz D A, Calzolari A, Buongiorno Nardelli M and Dougherty D B 2013 Modification of Molecular Spin Crossover in Ultrathin Films Nano Lett. 13 1429-34 
[12] Mahfoud T, Molnár G, Bonhommeau S, Cobo S, Salmon L, Demont P, Tokoro H, Ohkoshi S, Boukheddaden K and Bousseksou A 2009 Electric-Field-Induced ChargeTransfer Phase Transition: A Promising Approach Toward Electrically Switchable Devices J. Am. Chem. Soc. 131 15049-54

[13] Mahfoud T, Molnár G, Cobo S, Salmon L, Thibault C, Vieu C, Demont P and Bousseksou A 2011 Electrical properties and non-volatile memory effect of the $\left[\mathrm{Fe}\left(\mathrm{HB}(\mathrm{pz})_{3}\right)_{2}\right]$ spin crossover complex integrated in a microelectrode device Appl. Phys. Lett. 99053307

[14] Prins F, Monrabal-Capilla M, Osorio E A, Coronado E and van der Zant H S J 2011 Room-Temperature Electrical Addressing of a Bistable Spin-Crossover Molecular System Adv. Mater. 23 1545-9

[15] Lefter C, Davesne V, Salmon L, Molnár G, Demont P, Rotaru A and Bousseksou A 2016 Charge Transport and Electrical Properties of Spin Crossover Materials: Towards Nanoelectronic and Spintronic Devices Magnetochemistry 218

[16] Smullen C W, Nigam A Gurumurthi, S, Stan, M R 2011 The STeTSiMS STT-RAM simulation and modeling system, 2011 IEEE/ACM International Conference on Computer-Aided Design (ICCAD), pp. 318-325, IEEE Explore, INSPEC Accession Number: 12437597

[17] Wang R, Jiang L, Zhang Y, Wang L, Yang J 2015 Selective restore: An energy efficient read disturbance mitigation scheme for future STT-MRAM; Published in: 2015 52nd ACM/EDAC/IEEE Design Automation Conference (DAC), IEEE Xplore, INSPEC Accession Number: 15311851

[18] Chun K C, Zhao H, Harms J D, Kim T-H, Wang J-P, Kim C H 2013 A Scaling Roadmap and Performance Evaluation ofIn-Plane and Perpendicular MTJ Based STT-MRAMsfor High-Density Cache Memory IEEE J. Solid-State Circuits 48598

[19] Jiang X, Hao G, Wang X, Mosey A, Zhang X, Yu L, Yost A J, Zhang X, DiChiara A D, N'Diaye A T, Cheng X, Zhang J, Cheng R, Xu X and Dowben P A 2019 Tunable spinstate bistability in a spin crossover molecular complex J. Phys. Condens. Matter 31 315401

[20] Bogani L and Wernsdorfer W 2008 Molecular spintronics using single-molecule magnets Nat. Mater. 7 179-86 
[21] Christou G, Gatteschi D, Hendrickson D N and Sessoli R 2000 Single-Molecule Magnets MRS Bull. 25 66-71

[22] Lis T 1980 Preparation, structure, and magnetic properties of a dodecanuclear mixedvalence manganese carboxylate Acta Crystallogr. Sect. B Struct. Crystallogr. Cryst.

Chem. 36 2042-6

[23] Caneschi A, Gatteschi D, Sessoli R, Barra A L, Brunel L C, Guillot M 1991 Alternating current susceptibility, high field magnetization, and millimeter band EPR evidence for a ground $\mathrm{S}=10$ state in $\left[\mathrm{Mn}_{12} \mathrm{O}_{12}\left(\mathrm{CH}_{3} \mathrm{COO}\right)_{16}\left(\mathrm{H}_{2} \mathrm{O}\right)_{4}\right] \cdot 2 \mathrm{CH}_{3} \mathrm{COOH} .4 \mathrm{H}_{2} \mathrm{O}$ J. Am. Chem. Soc. 113 5873. doi:10.1021/ja00015a057.

[24] Sessoli R, Tsai, H L, Schake A R, Wang S, Vincent J B, Folting K, Gatteschi D, Christou G, Hendrickson D N 1993 High-spin molecules: [ $\left.\mathrm{Mn}_{12} \mathrm{O}_{12}\left(\mathrm{O}_{2} \mathrm{CR}\right)_{16}\left(\mathrm{H}_{2} \mathrm{O}\right)_{4}\right]$ J. Am. Chem. Soc.115 1804-1816; doi:10.1021/ja00058a027

[25] Sessoli R, Gatteschi D, Canesch A, Novak M A 1993 Magnetic bistability in a metal-ion cluster. Nature. 365 141-143; doi:10.1038/365141a0. ISSN 0028-0836.

[26] Guo, F.-S., Day, B. M., Chen, Y.-C., Tong, M.-L., Mansikkamäki, A., \& Layfield, R. A. (2018). Magnetic hysteresis up to 80 kelvin in a dysprosium metallocene single-molecule magnet. Science, 362 (6421), 1400-1403. doi:10.1126/science.aav0652

[27] Perrin M L, Burzurí E and van der Zant H S J 2015 Single-molecule transistors Chem. Soc. Rev. $44902-19$

[28] Natalya A. Zimbovskaya, 2013, Transport Properties of Molecular Junctions. Springer Tracts in Modern Physics 254 (Heidelberg)

[29] Nolting W, Ramakanth A 2009 Quantum Theory of Magnetism. Springer-Verlag (Heidelberg), ISBN 978-3-540-85415-9

[30] Gütlich P, Goodwin H A 2004 Spin Crossover in Transition Metal Compounds I. Springer-Verlag (Heidelberg), ISBN 3-540-40394-9

[31] Gütlich P, Garcia Y and Goodwin H A 2000 Spin crossover phenomena in Fe(ii) complexes Chem. Soc. Rev. 29 419-27

[32] Cambi L, Szegö L 1931 Ber. Deutsch Chem. Ges. 64167

[33] Cambi L, Malatesta L 1937 Ber. Deutsch Chem. Ges. 702067 
[34] Kyatskaya S, Galán Mascarós J R, Bogani L, Hennrich F, Kappes M, Wernsdorfer W and Ruben M 2009 Anchoring of Rare-Earth-Based Single-Molecule Magnets on SingleWalled Carbon Nanotubes J. Am. Chem. Soc. 131 15143-51

[35] Friedman J R, Sarachik M P, Tejada J and Ziolo R 1996 Macroscopic Measurement of Resonant Magnetization Tunneling in High-Spin Molecules Phys. Rev. Lett. 76 3830-3

[36] Thomas L, Lionti F, Ballou R, Gatteschi D, Sessoli R and Barbara B 1996 Macroscopic quantum tunnelling of magnetization in a single crystal of nanomagnets Nature 383 145-7

[37] Park H, Lim A K L, Alivisatos A P, Park J and McEuen P L 1999 Fabrication of metallic electrodes with nanometer separation by electromigration Appl. Phys. Lett. 75 301-3

[38] Amandine Bellec, Jerome Lagoute, Vincent Repain, 2018 Molecular electronics: Scanning tunneling microscopy and single-molecule devices Comptes Rendus Chimie 21 (2018) $1287 \mathrm{e} 1299$

[39] Ajuan Cui, Huanli Dong, and Wenping Hu 2015 Nanogap Electrodes towards Solid State Single-Molecule Transistors. Small 2015, 11, No. 46, 6115-6141

[40] Guo X, editor, 2019 Molecular-Scale Electronics, Topics in Current Chemistry Collections, Springer (heidelberg) ISBN: 978-3-030-03304-0

[41] Xiao J Dowben P A 2009 Changes in the adsorbate dipole layer with changing d-filling of the metal (II) (Co, Ni, Cu) phthalocyanines on $\mathrm{Au}(111)$ J. Phys.: Condens. Matter 21 052001

[42] Ishii H, Sugiyama K, Ito E and Seki K 1999 Energy level alignment and interfacial electronic structures at organic/metal and organic/organic interfaces Adv. Mater. 11605

[43] Novak M A, Sessoli R, Gatteschi D and Caneschi A 1993 Magnetic bistability in a metalion cluster Nature 365 141-3

[44] Gatteschi D, Sessoli R 2003 Quantum Tunneling of Magnetization and Related Phenomena in Molecular Materials. Angewandte Chemie International Edition 42 268297. doi:10.1002/anie.200390099

[45] Heersche H B, de Groot Z, Folk J A, van der Zant H S J, Romeike C, Wegewijs M R, Zobbi L, Barreca D, Tondello E and Cornia A 2006 Electron Transport through Single Mn12 Molecular Magnets Phys. Rev. Lett. 96206801

[46] Jo M-H, Grose J E, Baheti K, Deshmukh M M, Sokol J J, Rumberger E M, Hendrickson 
D N, Long J R, Park H and Ralph D C 2006 Signatures of Molecular Magnetism in Single-Molecule Transport Spectroscopy Nano Lett. 6 2014-20

[47] Zyazin A S, van den Berg J W G, Osorio E A, van der Zant H S J, Konstantinidis N P, Leijnse M, Wegewijs M R, May F, Hofstetter W, Danieli C and Cornia A 2010 Electric Field Controlled Magnetic Anisotropy in a Single Molecule Nano Lett. 10 3307-11

[48] Burzurí E, Zyazin A S, Cornia A and van der Zant H S J 2012 Direct Observation of Magnetic Anisotropy in an Individual $\mathrm{Fe}_{4}$ Single-Molecule Magnet Phys. Rev. Lett. 109 147203

[49] Park J, Pasupathy A N, Goldsmith J I, Chang C, Yaish Y, Petta J R, Rinkoski M, Sethna J P, Abruña H D, McEuen P L and Ralph D C 2002 Coulomb blockade and the Kondo effect in single-atom transistors Nature 417 722-5

[50] Liang W, Shores M P, Bockrath M, Long J R and Park H 2002 Kondo resonance in a single-molecule transistor Nature 417 725-9

[51] Meded V, Bagrets A, Fink K, Chandrasekar R, Ruben M, Evers F, Bernand-Mantel A, Seldenthuis J S, Beukman A and van der Zant H S J 2011 Electrical control over the Fe(II) spin crossover in a single molecule: Theory and experiment Phys. Rev. B 83245415

[52] Vincent R, Klyatskaya S, Ruben M, Wernsdorfer W and Balestro F 2012 Electronic readout of a single nuclear spin using a molecular spin transistor Nature 488 357-60

[53] Thiele S, Balestro F, Ballou R, Klyatskaya S, Ruben M and Wernsdorfer W 2014 Electrically driven nuclear spin resonance in single-molecule magnets Science 344 1135-8

[54] Ganzhorn M, Klyatskaya S, Ruben M and Wernsdorfer W 2013 Strong spin-phonon coupling between a single-molecule magnet and a carbon nanotube nanoelectromechanical system Nat. Nanotechnol. 8 165-9

[55] Lumetti S, Candini A, Godfrin C, Balestro F, Wernsdorfer W, Klyatskaya S, Ruben M and Affronte M 2016 Single-molecule devices with graphene electrodes Dalt. Trans. 45 $16570-4$

[56] Godfrin C, Ferhat A, Ballou R, Klyatskaya S, Ruben M, Wernsdorfer W, F. Balestro F 2017 Operating Quantum States in Single Magnetic Molecules: Implementation of Grover's Quantum Algorithm. Phys. Rev. Lett. 119187702

[57] Hao G, Mosey A, Jiang X, Yost A J, Sapkota K R, Wang G T, Zhang X, Zhang J, N'Diaye A T, Cheng R, Xu X and Dowben P A 2019 Nonvolatile voltage controlled 
molecular spin state switching Appl. Phys. Lett. 114032901

[58] Aravena D and Ruiz E 2012 Coherent transport through spin-crossover single molecules J. Am. Chem. Soc. 134 777-9

[59] Zapf V S, Sengupta P, Batista C D, Nasreen F, Wolff-Fabris F, Paduan-Filho A 2011 Magnetoelectric effects in an organometallic quantum magnet Phys. Rev. B 83 140405(R)

[60] Zapf V S, Kenzelmann M, Wolff-Fabris F, Balakirev F, Chen Y 2010 Magnetically induced electric polarization in an organometallic magnet Phys. Rev. B 82 060402(R)

[61] Chikara S, Gu J, Zhang X-G, Cheng H-P, Smythe N, Singleton J, Scott B, Krenkel E, Eckert J, Zapf V S 2019 Magnetoelectric behavior via a spin state transition Nature Commun. 10:4043 | https://doi.org/10.1038/s41467-019-11967-3 |

[62] Zhao W, Zou D, Sun Z, Xu Y, Ji G, Yu Y, Yang C 2019 Spin Logic Gates Operated by Protonation and Magnetism in Molecular Combinational Circuits Adv. Theory Simul. 2 1900057

[63] Zhang X, Palamarciuc T, Létard J-F, Rosa P, Lozada E V, Torres F, Rosa L G, Doudin B and Dowben P A 2014 The spin state of a molecular adsorbate driven by the ferroelectric substrate polarization Chem. Commun. 502255

[64] El Abbassi M, Sangtarash S, Liu X, Perrin M L, Braun O, Lambert C, van der Zant H S J, Yitzchaik S, Decurtins S, Liu S-X, Sadeghi H, Calame M 2019, Robust graphene-based molecular devices. Nature Nanotechnology 14 957-961 